CORRIGENDUM

doi:10.1038/nature10185

\title{
Imprints of fast-rotating massive stars in the Galactic Bulge
}

Cristina Chiappini, Urs Frischknecht, Georges Meynet, Raphael Hirschi, Beatriz Barbuy, Marco Pignatari, Thibaut Decressin \& André Maeder

Nature 472, 454-457 (2011)

In Table 1 of this Letter, rows 9 and $10([\mathrm{Y} / \mathrm{Fe}]$ and $[\mathrm{Sr} / \mathrm{Fe}])$ were inadvertently switched for stars number 2,3 and 4 . In addition, the [Y/Fe] value for star number 3 should be +1.50 and not +1.55 . The correct Table 1 is shown below, and this also now includes the $[\mathrm{Fe} / \mathrm{H}]$ values for each of the studied stars (taken from ref. 1 (ref. 4 in the original Letter)). The typical uncertainty in the $[\mathrm{Fe} / \mathrm{H}] \mathrm{values}$ is 0.2 dex. Table 1 has been corrected in the HTML and PDF version of the manuscript.

1. Barbuy, B. etal. VLT-FLAMES analysis of eight giants in the bulge metal-poor globular cluster NGC 6522: oldest cluster in the Galaxy? Astron. Astrophys. 507, 405-415 (2009).

Table 1 | Abundances of the eight stars in NGC 6522.

\begin{tabular}{|c|c|c|c|c|c|c|c|c|c|}
\hline Element & Reference & B-8 (star 1) & B-107 (star 2) & B-108 (star 3) & B-118 (star 4) & B-122 (star 5) & B-128 (star 6) & B-130 (star 7) & F-121 (star 8) \\
\hline$[\mathrm{Fe} / \mathrm{H}]$ & 4 & -1.03 & -1.11 & -1.10 & -0.84 & -0.87 & -0.79 & -1.09 & -1.15 \\
\hline$[\mathrm{O} / \mathrm{Fe}]$ & 4 & +0.25 & +0.50 & +0.70 & +0.30 & +0.70 & - & +0.50 & +0.50 \\
\hline$[\mathrm{Mg} / \mathrm{Fe}]$ & 4 & +0.10 & +0.27 & +0.33 & +0.20 & +0.20 & +0.25 & +0.40 & +0.40 \\
\hline$[\mathrm{Si} / \mathrm{Fe}]$ & 4 & +0.34 & +0.20 & +0.20 & +0.29 & +0.13 & +0.24 & +0.35 & +0.27 \\
\hline$[\mathrm{Ca} / \mathrm{Fe}]$ & 4 & +0.15 & +0.04 & +0.18 & +0.21 & +0.21 & +0.16 & +0.23 & +0.16 \\
\hline [Ti/Fe] & 4 & +0.12 & +0.14 & +0.21 & +0.11 & +0.19 & +0.17 & +0.21 & +0.16 \\
\hline$[\mathrm{Ba} / \mathrm{Fe}]$ & 4 & +0.95 & +0.50 & 0.00 & +1.00 & +0.60 & +0.90 & +0.25 & -0.25 \\
\hline$[\mathrm{La} / \mathrm{Fe}]$ & 4 & +0.50 & +0.50 & +0.30 & +0.50 & +0.30 & - & - & 0.00 \\
\hline$[\mathrm{Y} / \mathrm{Fe}]$ & This work & +1.20 & +1.00 & +1.50 & +1.50 & +1.20 & +1.50 & +1.20 & +1.20 \\
\hline [Sr/Fe] & This work & +1.20 & +1.30 & +1.00 & +0.50 & +0.50 & +1.50 & - & - \\
\hline$[\mathrm{Eu} / \mathrm{Fe}]$ & 4 & +0.50 & 0.00 & +0.50 & +0.50 & +0.30 & 0.00 & +0.80 & +0.50 \\
\hline$[\mathrm{Na} / \mathrm{Fe}]$ & 4 & +0.35 & -0.30 & -0.15 & +0.10 & +0.15 & +0.10 & +0.15 & -0.10 \\
\hline$[\mathrm{C} / \mathrm{Fe}]$ & This work & $\lesssim 0$ & $\lesssim 0$ & $\lesssim 0$ & $\lesssim 0$ & $\lesssim 0$ & $\lesssim 0$ & $\lesssim 0$ & $\lesssim 0$ \\
\hline
\end{tabular}

